\title{
A EDUCAÇÃO À DISTÂNCIA NA EDUCAÇÃO PERMANENTE DOS PROFISSIONAIS DA SAÚDE: REVISÃO
}

\section{LA EDUCACIÓN A DISTANCIA EN LA EDUCACIÓN PERMANENTE DE LOS PROFESIONALES DE SALUD: REVISIÓN}

\section{THE DISTANCE EDUCATION TO PROMOTE CONTINUOUS LEARNING OF HEALTH PROFESSIONALS: REVIEW}

\author{
Lívia Lima Ferraz ${ }^{1}$
}

\section{RESUMO}

A associação da necessidade de uma educação permanente com as tecnologias da informação e comunicação disponíveis e a flexibilização da Educação a Distancia obtiveram como resultado a inclusão do trabalho como um lugar importante para aprendizagem ${ }^{(1)}$. A educação permanente é uma necessidade premente para os profissionais da saúde, para que eles desenvolvam uma postura crítica, autoavaliativa, autoformadora e autogestora de seu aprendizado ${ }^{(2)}$. Essas características vão ao encontro do perfil de aluno ideal para Educação a Distancia. A metodologia deste estudo é de caráter bibliográfico. E o objetivo é reunir fundamentos e resultados que consubstancialize a implantação da Educação a Distância na Educação Permanente dos profissionais da saúde a partir de dois pontos relevantes: Incentivos/Políticas que promovam a implantação e Contribuições da Educação a Distância na Educação Permanente dos profissionais da saúde. Foi possível constatar que a modalidade de Ensino a Distância surge como uma estratégia importante e como uma facilitadora da Educação Permanente, principalmente, por conseguir estimular características relevantes para o pessoal da saúde e por conseguir romper barreiras territoriais e temporais.

Descritores: Educação a Distância, Políticas Públicas, Educação Continuada

\footnotetext{
${ }^{1}$ livia28ferraz@gmail.com
} 


\title{
RESUMEN
}

La asociación de la necesidad de una educación permanente con las tecnologías de información y comunicación disponibles y la flexibilidad de la Educación a Distancia obtuvieran como efecto la inserción del trabajo como un hogar importante para aprendizaje ${ }^{(1)}$. La Educación Permanente es una necesidad imprescindible para los profesionales de salud, para que ellos desarrollen una posición crítica y auto evaluativa ${ }^{(2)}$. Son estas características esenciales y coincidentes de los alumnos de la Educación a Distancia. La metodología deste estudio es de carácter bibliográfico. Y el objetivo es congregar fundamentos y resultados que apoye e base lo empleo de la Educación a Distancia en la Educación Permanente de lo personal de salud a partir de dos puntos relevantes: Incentivos/Políticas que fomenten su aplicación y las colaboraciones de la Educación a Distancia en educación permanente de los profesionales de salud. Fue posible constatar que la modalidad de enseñanza a distancia aparece como una estrategia importante y como una facilitadora de la Educación Permanente, principalmente, por lograr estimular características relevantes para el personal de salud y romper barreras territoriales y de tiempo.

Descriptores: Educación a Distancia, Políticas Pública, Educación Continua.

\begin{abstract}
The results of many articles and researches showed that employment have an important role for continuous learning. The main factors that made possible this continuous education were: the technology information advanced and distance education flexibilities. The evolution of on-line continuing education helps the health care professionals development many fundamental learning skills as self-assessment and self-criticism. Therefore, this articles objective is to identify how public policies could promote continuous learning of health professionals through distance education (DE) and the contributions of this education format for transformation health activities. In conclusion, the results were that distance education (DE) was an important strategy for permanent education, because (DE) developments good skills of learning and breaks territories barriers. Wherefore, distance education became an effective learning format.
\end{abstract}

Key words: Distance Education, Public Policies, Continuous Education 


\section{INTRODUÇÃO}

Os profissionais da área da saúde, com o advento tecnológico contemporâneo, possuem a oportunidade de estarem, permanentemente, informados e conscientes das inovações e descobertas. Um profissional atualizado e em atualização contínua cumpre o seu papel com a ética característica do setor da saúde ${ }^{(3)}$. Essa evolução tecnológica e a difusão de ferramentas digitais proporcionam o acesso às informações e a interatividade entre indivíduos de diferentes localidades geográficas, em tempo real ${ }^{(4)}$.

A associação da necessidade de uma educação permanente com as tecnologias da informação e comunicação disponíveis e a flexibilização da Educação a Distancia obtiveram como resultado a inclusão do trabalho como um lugar importante para aprendizagem ${ }^{(5)}$. É de suma importância salientar que a inclusão das tecnologias da informação e comunicação, tem por intuito o enriquecimento e a ampliação das práticas desses profissionais, a sua educação permanente e a sua participação social ${ }^{(3)}$. Assim, a educação a distância ganhou destaque por atender as demandas atuais de formação e qualificação e assumiu um papel importante na capacitação desses profissionais, contribuindo para a disseminação da informação e a melhoria na qualidade do atendimento na área da saúde. ${ }^{(4,6-7)}$

Maftum e Campos relatam em seu trabalho as suas experiências com a Educação a Distancia, e afirmam que a proposta atual diferencia-se pela conjugação da Educação a Distancia com metodologias ativas e composições de grupos multiprofissionais que permitem compartilhar com diferentes classes da área da saúde fazendo presente o tempo todo o exercício da interdisciplinaridade ${ }^{(7)}$. E, Garanhani et al. destacam que a forma de estudos na Educação a Distância exige algumas mudanças de hábito, especialmente, àquelas relacionadas à autodisciplina, à organização do tempo e à motivação para o aperfeiçoamento profissional (8).

O Ministério da Saúde, em sua publicação referente à Política Nacional de Educação Permanente em Saúde, ressalta que "Hoje em dia já é possível potencializar a Educação Permanente e em Serviço com os aportes das tecnologias de Educação a Distância. Em lugar de opor uma modalidade à outra, trata-se de enriquecer os projetos integrando ambas as contribuições, ou seja, faz-se necessário fortalecer os processos de Educação Permanente com a inclusão de aportes da Educação à distância, aproximando o conhecimento elaborado às práticas das equipes, alimentando suas contribuições no caminho de um progresso 
construtivo e inclusivo. Para isso, faz-se necessário o fortalecimento dos modelos educativos à distância privilegiando a problematização e integrando-os ao desenvolvimento de projetos de Educação Permanente em serviço" ${ }^{(9)}$.

Oliveira em seu estudo realça que a educação permanente é uma necessidade premente para os profissionais da saúde, para que eles desenvolvam uma postura crítica, autoavaliativa, autoformadora e autogestora de seu aprendizado. Essas características vão ao encontro do perfil de aluno ideal para Educação a Distância ${ }^{(2)}$.

\section{METODOLOGIA}

A metodologia deste estudo é de cunho bibliográfico. A pesquisa foi realizada por meio de informações e dados coletados de artigos publicados, e identificados a partir das bases de dados do Scientific Electronic Library Online - Scielo. Os principais descritores utilizados na busca foram: "Educação a Distância", "Educação Permanente", "Educação Continuada" e "Profissionais da saúde". A coleção de publicações da Biblioteca do Ministério da Saúde também foi consultada, a pesquisa usou palavras-chave, como: "política nacional" e “educação permanente".

\section{OBJETIVO}

Este artigo possui por objetivo reunir fundamentos e resultados que consubstancialize a implantação da Educação a Distância na Educação Permanente dos profissionais da saúde a partir de dois pontos relevantes.

- Incentivos/Políticas que promovam a implantação.

- Contribuições da Educação a Distância na Educação Permanente dos profissionais da saúde.

\section{Políticas Públicas x Educação Permanente em Saúde x Educação a Distância}

É possível afirmar que a Educação Permanente assumiu a posição de política pública na área da saúde a partir da difusão da Organização Pan-Americana de Saúde da proposta de Educação Permanente do Pessoal da Saúde para o desenvolvimento dos sistemas de saúde ${ }^{(1)}$. Em 2004, por meio da Portaria n 198/GM, o Ministério as Saúde instituiu a Política Nacional 
de Educação Permanente em Saúde como estratégia do Sistema Único de Saúde — SUS para formação e o desenvolvimento de trabalhadores para o setor ${ }^{(10)}$. Em 2007, houve, efetivamente, a sua implementação com o advento da publicação de suas diretrizes com a Portaria GM/MS n $1996^{(11)}$. Essas ações visam transformar e qualificar os profissionais atuantes da respectiva área.

A Portaria no 198/GM. definiu educação permanente: "é aprendizagem no trabalho, onde o aprender e o ensinar se incorporam ao cotidiano das organizações e ao trabalho. A educação permanente se baseia na aprendizagem significativa e na possibilidade de transformar as práticas profissionais" ${ }^{(10)}$.

Sob essa perspectiva educacional, o principal ponto no processo de ensino desses trabalhadores é que a aprendizagem seja significativa, ou seja, que para eles o conteúdo aprendido tenha alguma aplicação no seu contexto. A referida forma de aprendizagem tem o professor como um facilitador do processo de ensino-aprendizagem, nesse contexto, o seu papel é estimular o aluno a assumir uma postura crítica, ativa e reflexiva durante a construção do conhecimento ${ }^{(6)}$.

Um dos eixos de ação definidos pelo o Ministério da Saúde é a dinamização de recursos para a utilização da Educação a Distância como tecnologia pedagógica para a Educação Permanente ${ }^{(9)}$. A Educação a Distância possui vantagens únicas, inclusive quanto ao papel social, porque ela contribui para a disseminação de informações e melhoria na qualidade do atendimento na saúde ${ }^{(12)}$.

\section{Estratégia: Educação a Distância}

A Educação a Distância atende as especificidades dos profissionais da saúde e ela é mais que uma alternativa, ela é uma estratégia eficaz que atende a necessidade de universalização do acesso a uma educação de qualidade ${ }^{(13)}$. Ela facilita a autoaprendizagem, com a ajuda de recursos didáticos organizados e seus diversos suportes de informação e comunicação $^{(2)}$.

Sob a ótica brasileira, a Educação a Distância ganhou maior destaque e reconhecimento com a Nova Lei de Diretrizes e Bases da Educação Nacional — Lei $\mathrm{n}^{\circ}$ 9394/1996 -, “A educação à distância, organizada com abertura e regime especiais, será oferecida por instituições especificamente credenciadas pela União." (14) . A partir dessa lei, a Educação a Distância passou a fazer parte do Sistema Educacional Nacional e posteriores 
regulamentações sugiram a fim de consolidá-la, como a Portaria Ministerial nº 4.361, de 29 de dezembro de 2004 e o Decreto $\mathrm{n}^{\mathrm{o}} 5.622$, de 19 de dezembro de $2005^{(2,15-16)}$

Em 2002, aconteceu o Seminário Internacional - Política de Recursos Humanos em Saúde. O evento foi organizado pelo Ministério da Saúde e pela Organização Pan-Americana da Saúde. Uma de suas proposições definiu que para a Educação Permanente deveria haver investimentos em tecnologia de educação a distância, apoiando a construção de salas de teleconferências ${ }^{(17)}$.

Desta forma, constata-se que o governo tem promovido estratégias que impulsionem as diversas experiências institucionais de mudança e que reiterem a perspectiva da integralidade do cuidado, a centralidade do trabalho em saúde como a base organizadora da formação profissional e que a Educação a Distância aparece como um eixo de ação na implantação da Educação Permanente em Saúde ${ }^{(9,18)}$.

Estudos entendem que a Educação Permanente pode ser realizada por meio da Educação a Distância, por ela oferecer a oportunidade para a produção do diálogo e proporcionar o estudo cooperativo entre os profissionais ${ }^{(2)}$. A Educação a Distância é proposta como um convite ao estudo e a indagação que conduzem ao pensamento crítico e reflexivo. E é esse convite que abre caminho para novos processos de pesquisa ${ }^{(19)}$. Assim, ela surge como ferramenta importante de sobrevivência pessoal e profissional ${ }^{(2)}$.

Nunes TWN, et al. em sua pesquisa verificaram que, apesar de algumas limitações, os alunos puderam interagir num espaço multiprofissional/interdisciplinar e demonstraram indícios de desequilíbrio, reflexões e mudança de postura necessários à cooperação e à prática integral em saúde $^{(20)}$. E, Oliveira MAN concluiu que, mesmo com os desafios a ser superados, a Educação a Distância, como modalidade de ensino e aprendizagem, é uma nova perspectiva para área de saúde que deve ser implementada ${ }^{(2)}$.

Deste modo, é possível verificar que, hoje, há bases legais e regulamentares que dão suporte a implementação tanto à Educação Permanente quanto à Educação a Distância. E, diversos estudos fundamentam como elas podem ser eficazes e importantes, em especial, para os profissionais do setor da saúde. Claro que existem obstáculos, como: escassez de tempo desses trabalhadores e dificuldade em usar as ferramentas e os ambientes virtuais ${ }^{(2)}$, há também preocupações como o desconhecimento dos muitos modos de aprender-ensinar que envolvem o uso de tecnologias nos processos educativos, inclusive as de comunicação e informação altamente desenvolvidas e diversificadas ${ }^{(18)}$. Entretanto, esses empecilhos podem 
ser superados por meio de um planejamento adequado, do diagnóstico do perfil dos usuários

${ }^{(13)}$ e de um sistema de preparação e acompanhamento de todos os envolvidos ${ }^{(21)}$.

\section{CONSIDERAÇÕES FINAIS}

O processo educacional do pessoal da saúde é construído por diversas ações encadeadas e orientadas a obter uma aprendizagem de acordo com a sua realidade laboral, com isso e com as constantes descobertas científicas, há a necessidade desse processo ser permanente, ao longo de toda a sua vida profissional ${ }^{(22)}$.

Devido a essa necessidade, políticas de âmbito nacional e regionais foram implementadas para atender esses profissionais. Desta forma, hoje, ela constitui um instrumento privilegiado de ampliação da qualidade da análise da realidade e da construção de ações de promoção da saúde e de cuidado, num contexto de democratização da gestão e das relações de trabalho ${ }^{(23)}$.

E, a modalidade de Ensino a Distância surge nesse contexto como um estratégia importante para que a classe da saúde alcance seus objetivos profissionais e supram suas carências educacionais e em que estão em frequente evolução. Assim, a Educação a Distância surge como um facilitador da Educação Permanente, principalmente, por conseguir estimular características relevantes para o pessoal da saúde e por conseguir romper barreiras territoriais e temporais.

\section{REFERÊNCIAS BIBLIOGRÁFICAS}

1- Laguardia Josué, Casanova Ângela, \& Machado Rejane. (2010). A experiência de aprendizagem on-line em um curso de qualificação profissional em saúde. Trabalho, Educação e Saúde, 8(1), 97-122.Available from: http://www.scielo.br/scielo.php?script=sci_arttext\&pid=S198177462010000100006\&lng=en\&tlng=pt. 10.1590/S1981-77462010000100006.

2- Oliveira, MAN. Educação à Distância como estratégia para a educação permanente em saúde: possibilidades e desafios. Rev. bras. enferm., Brasília, v. 60, n. 5, Oct. 2007. Available from:<http://www.scielo.br/scielo.php?script=sci_arttext\&pid=S003471672007000500019\&1 ng=en\&nrm=iso $>$. 
3- Cardoso Jefferson Paixão, Rosa Valéria Argolo, Lopes Claudia Ribeiro Santos, Vilela Alba Benemérita Alves, Santana Aurisan Souza de, Silva Sandro Tonini da. Construção de uma práxis educativa em informática na saúde para ensino de graduação. Ciênc. saúde coletiva [serial on the Internet]. 2008 Feb; 13(1): 283-288. Available from: http://www.scielo.br/scielo.php?script=sci_arttext\&pid=S1413-

$81232008000100031 \& \operatorname{lng}=$ en.

4- Silva Gizelda Monteiro da, Seiffert Otília Maria L. B.. Educação continuada em enfermagem: uma proposta metodológica. Rev. bras. enferm. [online]. 2009, vol.62, n.3, pp. 362-366.

Available from:<http://www.scielo.br/scielo.php?script=sci_arttext\&pid=S003471672009000300005\&1 $\mathrm{ng}=\mathrm{en} \& \mathrm{nrm}=\mathrm{iso}>$.

5- Cardoso, IM. "Rodas de educação permanente" na atenção básica de saúde: analisando contribuições. Saúde soc., São Paulo, 2013. Available from <http://www.scielo.br/scielo.php?script=sci_arttext\&pid=S010412902012000500002\&lng=en \&nrm=iso>.

6 - Neves-Junior Wellington Furtado Pimenta, Haddad Cecília Maria Kalil, Sousa Fernando Sequeira, Pisa Ivan Torres. Educação a Distância (EaD) em Física Médica. Interface (Botucatu) [serial on the Internet]. 2011; 15(39): 1199-1206. Available from: http://www.scielo.br/scielo.php?script=sci_arttext\&pid=S1414$32832011000400018 \& \operatorname{lng}=$ en.

7 - Maftum M, Campos, J. Capacitação Pedagógica Na Modalidade De Educação A Distância: Desafio para Ativar Processos de Mudança na Formação de Profissionais de Saúde. Cogitare Enfermagem, América do Norte, 13, ago. 2008. Disponível em: http://ojs.c3sl.ufpr.br/ojs2/index.php/cogitare/article/view/11973/8444

8- Garanhani ML, Ribeiro RP, Ortiz MCL. Educação à distância: uma ferramenta para educação permanente de enfermeiros que trabalham com assistência perioperatória. Cogitare Enfermagem. 2008 Out/Dez; 13(4): 558-65. Disponível em: http://ojs.c3sl.ufpr.br/ojs2/index.php/cogitare/article/view/13116/8874

9- Brasil. Ministério da Saúde. Secretaria de Gestão do Trabalho e da Educação na Saúde. Departamento de Gestão da Educação em Saúde. Política Nacional de Educação Permanente em Saúde / Ministério da Saúde, Secretaria de Gestão do Trabalho e da Educação na Saúde, Departamento de Gestão da Educação em Saúde. - Brasília: Ministério da Saúde, 2009. 64 p. - (Série B. Textos Básicos de Saúde) (Série Pactos pela Saúde 2006; v. 9) 
10- Brasil. Ministério Da Saúde. Portaria n.198/GM/MS, de 13 de fevereiro de 2004. Institui a Política Nacional de Educação Permanente em Saúde como estratégia do Sistema Único de Saúde para formação e o desenvolvimento de trabalhadores para o setor e dá outras providências. Diário Oficial da União, Brasília, DF, 16 fev. 2004. Seção 1.

11 - Brasil. Ministério Da Saúde. Portaria n.1996 GM/MS, de 20 de Agosto de 2007. Dispõe sobre as diretrizes para a implementação da Política Nacional de Educação Permanente em Saúde. Diário Oficial da União, Brasília, DF, 20 ago. 2007.

12- Neves-Junior WFP et al. Educação a Distância (EaD) em Física Médica. Interface (Botucatu), Botucatu, v. 15, n. 39, Dec. 2011. Available from $<$ http://www.scielo.br/scielo.php?script=sci_arttext\&pid=S141432832011000400018\&lng=en $\& n m=$ iso $>$.

13- Araujo E. Avaliação de Perfil de Profissionais da Saúde para Educação Distância Baseado na Lógica Difusa. XIII Congresso Brasileiro em Informática em Saúde - CBIS 2012.

14- Brasil. Lei n. 9.394, de 20 de dezembro de 1996. Estabelece as diretrizes e bases da educação nacional. Diário Oficial da União, Brasília, 20 dez. 1996. Disponível em: <www.planalto.gov.br/ccivil_03/Leis/L9394.htm>.

15- Brasil. Portaria Ministerial n. 4361, de 29 de dezembro de 2004. Diário Oficial da União, Brasília, 29 dez. 2004. Seção 1, p. 66-67.

16- Brasil. Decreto n. 5.622, de 19 de dezembro de 2005. Regulamenta o artigo 80 da Lei no 9.394, de 20 de dezembro de 1996, que estabelece as diretrizes e bases da educação nacional. Diário Oficial da União. Brasília, DF, 20 dez. 2005. Disponível em: <http://www.planalto.gov.br/ccivil_03/_Ato2004-2006/2005/Decreto/D5622.htm>.

17 - OPAS-OMS. Políticas de recursos humanos em saúde. Brasil. Ministério da Saúde.

Relatório Internacional. Brasília: Ministério da Saúde; 2002.

18- Torrez MNFB. Educação a distância e a formação em saúde: nem tanto, nem tão pouco. Trab. educ. saúde, Rio de Janeiro, v. 3, n. 1, Mar. 2005. Available from: <http://www.scielo.br/scielo.php?script=sci_arttext\&pid=S198177462005000100009\&lng=en $\&$ nrm $=$ iso $>$.

19- Brovelli, MS. La educación a distancia: una invitación a la construcción conjunta. Trab. educ. saúde [online]. 2005, vol.3, n.1.

20- Nunes TWN, Franco SRK; Silva VD da. Como a educação a distância pode contribuir para uma prática integral em saúde?. Rev. bras. educ. med., Rio de Janeiro, v. 34, n. 4, Dec. 
from: $<$ http://www.scielo.br/scielo.php?script=sci_arttext\&pid=S010055022010000400011\&l $\mathrm{ng}=\mathrm{en} \& \mathrm{nrm}=\mathrm{iso}>$.

21 - Gomes, RCG et. al. Tecnologia e Andragogia: aliadas na educação a distância - Tema: Gestão de Sistemas de Educação a Distância. Disponível em:

http://www2.abed.org.br/visualizaDocumento.asp?Documento_ID=84

22 - Chang MI, Mojica MJ, Haddad J. Proceso de Educación Permanente en Salud. Educ. Med. Salud, 1987; Vol. 21, n. 1.

23 - Tesser Charles Dalcanale, Garcia Adir Valdemar, Vendruscolo Carine, Argenta Cleonete Elena. Estratégia saúde da família e análise da realidade social: subsídios para políticas de promoção da saúde e educação permanente. Ciênc. saúde coletiva [serial on the Internet]. 2011 Nov 16(11):42954306. Available from: http://www.scielo.br/scielo.php?script=sci_arttext\&pid=S1413$81232011001200002 \& \operatorname{lng}=$ en. 